\title{
ON PROPERTIES OF SEMIPREINVEX FUNCTIONS
}

\author{
X.M. YANG, X.Q. YANG AND K.L. TeO
}

In this paper, we first discuss some basic properties of semipreinvex functions. We then show that the ratio of semipreinvex functions is semipreinvex, which extends earlier results by Khan and Hanson [6] and Craven and Mond [3]. Finally, saddle point optimality criteria are developed for a multiobjective fractional programming problem under semipreinvexity conditions.

\section{INTRODUCTION}

Let $\mathbb{R}^{n}$ denote an $n$-dimension Euclidean space. In [4], Hanson considered the real differentiable function $f(x)$ on $\mathbb{R}^{n}$ whose gradient $\nabla f(x)$ satisfies the condition: for any $x, y \in \mathbb{R}^{n}$, there exists a vector $\eta(x, y) \in \mathbb{R}^{n}$ such that

$$
f(y) \geqslant f(x)+\nabla f(x) \eta(x, y) .
$$

Craven [2] called this an invex function. Later, Weir and Mond [10] and Weir and Jeyakumar [9] introduced preinvex functions defined as follows.

Let $K \subset \mathbb{R}^{n}$ and $f: K \longrightarrow \mathbb{R}$. Then $f$ is preinvex if for any $x, y \in K$, there exists a vector $\eta(x, y) \in \mathbb{R}^{n}$, for all $\alpha \in[0,1], y+\alpha \eta(x, y) \in K$

$$
f(y+\alpha \eta(x, y)) \leqslant \alpha f(x)+(1-\alpha) f(y) .
$$

It is easy to show that preinvexity is a generalisation of invexity for nondifferentiable function.

In [11], Yang and Chen presented a wider class of generalised convex functions, called semipreinvex functions, which includes the classes of preinvex functions and arcconnected convex functions. The class of generalised convex functions preserves some nice properties that convex functions have (Noor [8]).

A set $K$ in $\mathbb{R}^{n}$ is said to satisfy the "semi-connected" property, if for any $x, y$ $\in K$ and $\alpha \in[0,1]$, there exists a vector $\eta(x, y, \alpha) \in \mathbb{R}^{n}$, such that $y+\alpha \eta(x, y, \alpha)$

Received 15th May, 2003

This research was partially supported by the National Natural Science Foundation of China, the Key Project of Chinese Ministry of Education, the Foundation of Chongqing Ministry of Education and Competitive Research Grants of The Hong Kong Polytechnic University.

Copyright Clearance Centre, Inc. Serial-fee code: 0004-9727/03 $\$ A 2.00+0.00$. 
$\in K$. Let $K$ be a set in $\mathbb{R}^{n}$ having the "semi-connected" property with $\eta(x, y, \alpha): K$ $\times[0,1] \longrightarrow \mathbb{R}^{n}$ and $f(x)$ be a real function on $K$. Then $f$ is called semi-preinvex with respect to $\eta(x, y, \alpha)$ if for $x, y \in K$ and $\alpha \in[0,1]$,

$$
f(y+\alpha \eta(x, y, \alpha)) \leqslant \alpha f(x)+(1-\alpha) f(y)
$$

holds and $\lim _{\alpha \downarrow 0} \alpha \eta(x, y, \alpha)=0$.

In this paper, we give some new properties of semipreinvex functions. In particular, we show that the ratio of two semipreinvex functions is a semipreinvex function. This result extends the property, that if $f$ and $-g$ are invex then $f / g$ is invex, obtained by Khan and Hanson [6] and Craven and Mond [3]. We also point out that a statement made by Noor in [8] is not correct. Also, saddle point optimality criteria involving semipreinvex functions are developed for a multiobjective fractional programming problem.

\section{SOME NEW PROPERTIES OF SEMIPREINVEX FUNCTIONS}

The following results characterise semipreinvex functions.

THEOREM 2.1. Let $K$ be a semi-connected set with respect to $\eta(x, y, \alpha)$. A function $f: K \longrightarrow \mathbb{R}$ is semipreinvex with respect to the same $\eta(x, y, \alpha)$ if and only if, for all $x, y \in K, \alpha \in[0,1]$, and $u, v \in \mathbb{R}$,

$$
f(x)<u \text { and } f(y)<v \quad \Longrightarrow \quad f(y+\alpha \eta(x, y, \alpha))<\alpha u+(1-\alpha) v .
$$

Proof: Let $f$ be semipreinvex with respect to $\eta$, and let $f(x)<u, f(y)<v$, $0<\alpha<1$. From the definition of semipreinvexity, we have

$$
f(y+\alpha \eta(x, y, \alpha)) \leqslant \alpha f(x)+(1-\alpha) f(y)<\alpha u+(1-\alpha) v .
$$

Conversely, let $x, y \in K, \alpha \in[0,1]$. For any $\delta>0, f(x)<f(x)+\delta, f(y)<f(y)+\delta$. By the assumption of the theorem, we have, for $0<\alpha<1$,

$$
f(y+\alpha \eta(x, y, \alpha))<\alpha(f(x)+\delta)+(1-\alpha)(f(y)+\delta)=\alpha f(x)+(1-\alpha) f(y)+\delta .
$$

Since $\delta>0$ can be arbitrarily small, it follows that

$$
f(y+\alpha \eta(x, y, \alpha)) \leqslant \alpha f(x)+(1-\alpha) f(y), \quad \alpha \in(0,1) .
$$

Hence, $f$ is semipreinvex on $K$. This completes the proof.

THEOREM 2.2. Let $K$ be a semi-connected set with respect to $\eta(x, y, \alpha)$. A function $f: K \longrightarrow \mathbb{R}$ is semipreinvex with respect to the same $\eta(x, y, \alpha)$ if and only if the set

$$
F(f)=\{(x, u): x \in K, u \in \mathbb{R}, f(x)<u\}
$$


is semi-connected with respect to $\eta_{1}: F(f) \times F(f) \times[0,1] \longrightarrow \mathbb{R}^{n+1}$, where

$$
\eta_{1}((y, v),(x, u), \alpha)=(\eta(y, x, \alpha), v-u)
$$

for all $(x, u),(y, v) \in F(f)$.

Proof: Necessity. Let $(x, u) \in F(f)$ and $(y, v) \in F(f)$, that is, $f(x)<u$ and $f(y)<v$. From the semipreinvexity of $f$, we have

$$
f(y+\alpha \eta(x, y, \alpha)) \leqslant(1-\alpha) f(x)+\alpha f(y)<(1-\alpha) u+\alpha v, \quad \alpha \in(0,1) .
$$

It follows that

$$
(x+\alpha \eta(y, x, \alpha),(1-\alpha) u+\alpha v) \in F(f), \quad \alpha \in(0,1) .
$$

That is,

$$
(x, u)+\alpha(\eta(y, x, \alpha), v-u) \in F(f), \alpha \in(0,1) .
$$

Hence, $F(f)$ is a semi-connected set with respect to $\eta_{1}((y, v),(x, u), \alpha)=(\eta(y, x, \alpha), v$ $-u)$.

Sufficiency. Assume that $F(f)$ is a semi-connected set with respect to

$$
\eta_{1}((y, v),(x, u), \alpha)=(\eta(y, x, \alpha), v-u) .
$$

Let $x, y \in K$ and $u, v \in R$ such that $f(x)<u, f(y)<v$. Then, $(x, u) \in F(f)$ and $(y, v)$ $\in F(f)$. From the semi-connectedness of the set $F(f)$ with respect to $\eta_{\mathrm{1}}((y, v),(x, u), \alpha)$ $=(\eta(y, x, \alpha), v-u)$, we have

$$
(x, u)+\alpha \eta_{1}((y, v),(x, u), \alpha) \in F(f), \quad \alpha \in(0,1) .
$$

It follows that

$$
(x+\alpha \eta(y, x, \alpha),(1-\alpha) u+\alpha v) \in F(f), \quad \alpha \in(0,1) .
$$

That is,

$$
f(y+\alpha \eta(x, y, \alpha))<\alpha u+(1-\alpha) v .
$$

Then, by Theorem $2.1, f$ is a semipreinvex function with respect to $\eta(x, y, \alpha)$ on $K$.

In Noor [8], the following statement is given: a function $f: K \rightarrow \mathbb{R}$ is semipreinvex with respect to $\eta(x, y, \alpha)$ if and only if the epigraph of $f$,

$$
G(f)=\{(x, u): x \in K, u \in \mathbb{R}, f(x) \leqslant u\}
$$

is semi-connected with respect to the same $\eta$. This statement contains an error, that is, the set $G(f)$ is semi-connected with respect to $\eta_{1}((y, v),(x, u), \alpha)=(\eta(y, x, \alpha), v-u)$, but not with respect to $\eta$. We give a correction of this statement below. 
THEOREM 2.3. Let $K$ be a semi-connected set with respect to $\eta(x, y, \alpha)$. A function $f: K \longrightarrow \mathbb{R}$ is semipreinvex with respect to the same $\eta(x, y, \alpha)$ if and only if the set

$$
G(f)=\{(x, u): x \in K, u \in \mathbb{R}, f(x) \leqslant u\}
$$

is a semi-connected set with respect to $\eta_{1}: G(f) \times G(f) \longrightarrow \mathbb{R}^{n+1}$, where

$$
\eta_{1}((y, v),(x, u), \alpha)=(\eta(y, x, \alpha), v-u),
$$

for all $(x, u),(y, v) \in G(f)$.

Proof: Necessity. Let $(x, u) \in G(f)$ and $(y, v) \in G(f)$, that is, $f(x) \leqslant u$ and $f(y) \leqslant v$. From the semipreinvexity of $f$, we have

$$
f(y+\alpha \eta(x, y, \alpha)) \leqslant(1-\alpha) f(x)+\alpha f(y) \leqslant(1-\alpha) u+\alpha v, \quad \alpha \in(0,1) .
$$

It follows that

$$
(x+\alpha \eta(y, x, \alpha),(1-\alpha) u+\alpha v) \in G(f) \alpha \in(0,1) .
$$

That is,

$$
(x, u)+\alpha(\eta(y, x, \alpha), v-u) \in G(f), \quad \alpha \in(0,1) .
$$

Hence, $G(f)$ is a semi-connected set with respect to $\eta_{1}((y, v),(x, u), \alpha)=(\eta(y, x, \alpha), v$ $-u)$.

Sufficiency. Assume that $G(f)$ is a semi-connected set with respect to

$$
\eta_{1}((y, v),(x, u), \alpha)=(\eta(y, x, \alpha), v-u) .
$$

Let $x, y \in K$ and $u, v \in R$ such that $f(x) \leqslant u, f(y) \leqslant v$. Then, $(x, u) \in G(f)$ and $(y, v)$ $\in G(f)$. From the semi-connectedness of the set $G(f)$ with respect to $\eta_{1}((y, v),(x, u), \alpha)$ $=(\eta(y, x, \alpha), v-u)$, we have

$$
(x, u)+\alpha \eta_{1}((y, v),(x, u), \alpha) \in G(f), \quad \alpha \in(0,1) .
$$

It follows that

$$
(x+\alpha \eta(y, x, \alpha),(1-\alpha) u+\alpha v) \in G(f), \quad \alpha \in(0,1) .
$$

That is,

$$
f(y+\alpha \eta(x, y, \alpha)) \leqslant \alpha u+(1-\alpha) v .
$$

Hence, $f$ is a semipreinvex function with respect to $\eta(x, y, \alpha)$ on $K$.

THEOREM 2.4. Let $K \subset \mathbb{R}^{n+1}$ and

$$
f(x)=\inf \{u: u \in R,(x, u) \in K\}, \forall x \in \mathbb{R}^{n} .
$$


If $K$ is a semi-connected set with respect to $\eta_{1}: K \times K \times[0,1] \longrightarrow \mathbb{R}^{n+1}$ and $\eta$ : $\mathbb{R}^{n} \times \mathbb{R}^{n} \times[0,1] \longrightarrow \mathbb{R}^{n}$ satisfying

$$
\eta_{1}((y, v),(x, u), \alpha)=(\eta(y, x, \alpha), v-u), \text { for all }(x, u),(y, v) \in K,
$$

then $f: \mathbb{R}^{n} \longrightarrow \mathbb{R}$ is a semipreinvex function with respect to $\eta$ on $\mathbb{R}^{n}$.

Proof: It suffices to show that the function $f: \mathbb{R}^{n} \longrightarrow \mathbb{R}$ is a semipreinvex function with respect to $\eta(y, x, \alpha)$. To see this, let $x, y \in \mathbb{R}^{n}$. Since $K$ is a semi-connected set with respect to $\eta_{1}((y, v),(x, u), \alpha)$, we have, for any $(x, u),(y, v) \in K$,

$$
(x, u)+\alpha \eta_{1}((y, v),(x, u), \alpha) \in K, \forall \alpha \in(0,1) .
$$

It follows from $\eta_{1}((y, v),(x, u), \alpha)=(\eta(y, x, \alpha), v-u)$ that

$$
(x, u)+\alpha \eta_{1}((y, v),(x, u), \alpha)=(x+\alpha \eta(x, y, \alpha),(1-\alpha) u+\alpha v) \in K, \quad \forall \alpha \in(0,1) .
$$

By the definition of $f$, we obtain

$$
f(x+\alpha \eta(x, y, \alpha)) \leqslant \alpha f(y)+(1-\alpha) f(x), \quad \forall \alpha \in(0,1) .
$$

Hence, $f$ is a semipreinvex function with respect to $\eta$ on $\mathbb{R}^{n}$.

THEOREM 2.5. Let $I$ be an index set. If $\left(S_{i}\right)_{i \in I}$ is a family of semi-connected subsets in $\mathbb{R}^{n+1}$ with respect to the same function $\eta_{1}: \mathbb{R}^{n+1} \times \mathbb{R}^{n+1} \times[0,1] \longrightarrow \mathbb{R}^{n+1}$, then their intersection $\bigcap_{i \in I} S_{i}$ is a semi-connected set with respect to the same function $\eta_{1}$.

Proof: Let $(x, \alpha),(y, \beta) \in \bigcap_{i \in I} S_{i}$. Then, for each $i \in I,(x, \alpha),(y, \beta) \in S_{i}$. Since $S_{i}$ is a semi-connected set with respect to the same function $\eta_{1}$, for each $i \in I$, it follows that

$$
\left(y+\alpha \eta_{1}(x, y, \alpha), \alpha \alpha+(1-\alpha) \beta\right) \in S_{i}, \quad 0 \leqslant \alpha \leqslant 1
$$

Thus

$$
\left(y+\alpha \eta_{1}(x, y, \alpha), \alpha \alpha+(1-\alpha) \beta\right) \in \bigcap_{i \in I} S_{i}, \quad \forall \alpha \in[0,1] .
$$

Hence, the result follows.

THEOREM 2.6. Let $K \subseteq \mathbb{R}^{n}$ be a semi-connected set with respect to

$$
\eta: \mathbb{R}^{n} \times \mathbb{R}^{n} \times[0,1] \longrightarrow \mathbb{R}^{n}
$$

and a family of real-valued functions $\left(f_{i}\right)_{i \in I}$ be semipreinvex with respect to the same $\eta$ and bounded from above on $K$. Then, the function $f(x)=\sup _{i \in I} f_{i}(x)$ is a semipreinvex function with respect to the same $\eta$ on $K$.

Proof: Since each $f_{i}$ is a semipreinvex function for the same function $\eta$ on $K$, it follows from Theorem 2.3 that its epigraph

$$
G\left(f_{i}\right)=\left\{(x, \alpha) \mid x \in K, \alpha \in \mathbb{R}, f_{i}(x) \leqslant \alpha\right\}
$$


is a semi-connected set in $\mathbb{R}^{n} \times \mathbb{R}$ with respect to $\eta_{1}=(\eta(y, x, \alpha), v-u)$. Therefore, their intersection

$$
\begin{aligned}
\bigcap_{i \in I} G\left(f_{i}\right) & =\left\{(x, \alpha) \mid x \in K, \alpha \in \mathbb{R}, f_{i}(x) \leqslant \alpha, i \in I\right\} \\
& =\{(x, \alpha) \mid x \in K, \alpha \in \mathbb{R}, f(x) \leqslant \alpha\}
\end{aligned}
$$

is also a semi-connected set in $\mathbb{R}^{n} \times \mathbb{R}$ with respect to $\eta_{1}=(\eta(y, x, \alpha), v-u)$, by Theorem 2.5. This intersection is the epigraph of $f$. Hence, by Theorem $2.3, f$ is a semipreinvex function with respect to $\eta$ on $K$.

The following theorem is due to Khan and Hanson [6] and Craven and Mond [3].

THEOREM 2.7. Let $X_{0} \subset \mathbb{R}^{n}$ and let $f$ and $g$ be real-valued functions defined on $X_{0}$. If $f(x) \geqslant 0, g(x)>0, f(x)$ and $-g(x)$ are invex with respect to the same $\eta(x, y)$ on $X_{0}$, then $f(x) / g(x)$ is an invex function with respect to $\bar{\eta}(x, y)=(g(y) / g(x)) \eta(x, y)$.

Now we present a similar result in:

THEOREM 2.8. Let $X_{0} \subset \mathbb{R}^{n}$ and let $f$ and $g$ be real-valued functions defined on $X_{0}$. If $f(x) \geqslant 0, g(x)>0, f(x)$ and $-g(x)$ are semipreinvex with respect to a same $\eta(x, y, \alpha)$ on $X_{0}$, then $f(x) / g(x)$ is a semipreinvex function with respect to $\bar{\eta}(x, y, \alpha)$ $=(g(y)) /(\alpha g(y)+(1-\alpha) g(x)) \eta(x, y, \alpha)$.

Proof: Since $f(x)$ and $-g(x)$ are semipreinvex functions with respect to the same $\eta(x, y, \alpha)$ and $f(x) \geqslant 0, g(x)>0$, we have, for any $x, y \in X_{0}$, and $\alpha \in[0,1]$,

$$
\begin{aligned}
\left(\frac{f}{g}\right)(y & +\alpha \bar{\eta}(x, y, \alpha))=\frac{f(y+\alpha \bar{\eta}(x, y, \alpha))}{g(y+\alpha \bar{\eta}(x, y, \alpha))} \\
& =\frac{f(y+\alpha(g(y)) /(\alpha g(y)+(1-\alpha) g(x)) \eta(x, y, \alpha))}{g(y+\alpha(g(y)) /(\alpha g(y)+(1-\alpha) g(x)) \eta(x, y, \alpha))} \\
& \leqslant \frac{(\alpha g(y)) /(\alpha g(y)+(1-\alpha) g(x)) f(x)+(1-(\alpha g(y)) /(\alpha g(y)+(1-\alpha) g(x))) f(y)}{(\alpha g(y)) /(\alpha g(y)+(1-\alpha) g(x)) g(x)+(1-(\alpha g(y)) /(\alpha g(y)+(1-\alpha) g(x))) g(y)} \\
& =\alpha \frac{f(x)}{g(x)}+(1-\alpha) \frac{f(y)}{g(y)}=\alpha\left(\frac{f}{g}\right)(x)+(1-\alpha)\left(\frac{f}{g}\right)(y)
\end{aligned}
$$

That is, $(f(x)) /(g(x))$ is a semipreinvex function with respect to $\bar{\eta}(x, y, \alpha)$.

From Theorem 2.8, it is easy to prove the following result.

THEOREM 2.9. Let $x_{0} \subset \mathbb{R}^{n}$, and let $f$ and $g$ be real-valued differentiable functions defined on $X_{0}$. If $f(x) \geqslant 0, g(x)>0, f(x)$ and $-g(x)$ are semipreinvex with respect to the same $\eta(x, y, \alpha)$ on $X_{0}$, and $\lim _{\alpha \rightarrow 0} \eta(x, y, \alpha)=\eta(x, y)$, then $(f(x)) /(g(x))$ is an invex function with respect to

$$
\bar{\eta}(x, y)=\frac{g(y)}{g(x)} \eta(x, y) .
$$

In particular, we wish to point out that the following question remains open: Is there a similar result as that of Theorem 2.9 for preinvex functions? 


\section{APPLICATIONS TO MULTIOBJECTIVE FRACTIONAL PROGRAMMING}

The following notations for vector orderings in $\mathbb{R}^{n}$ will be used:

$$
\begin{array}{ll}
x>y \quad \text { if and only if } \quad x_{i}>y_{i}, i=1,2, \ldots, n ; \\
x \geqq y \quad \text { if and only if } \quad x_{i} \geqslant y_{i}, i=1,2, \ldots, n ; \\
x \geqslant y \quad \text { if and only if } \quad x_{i} \geqslant y_{i}, i=1,2, \ldots, n, \text { but } x \neq y ; \\
x \ngtr y \quad \text { is the negation of } \quad x \geqslant y ; \\
x \ngtr y \quad \text { is the negation of } \quad x>y ;
\end{array}
$$

Multiobjective fractional programming problems have been studied by many authors. In this section, we obtain saddle point optimality criteria and Lagrangian type duality results for multiobjective fractional programming problems involving semipreinvex functions. We consider the following problem.

Primal Problem (FP):

$$
\begin{array}{ll}
\text { Minimise } & \frac{f(x)}{g(x)}:=\left(\frac{f_{1}(x)}{g_{1}(x)}, \ldots, \frac{f_{k}(x)}{g_{k}(x)}\right) \\
\text { subject to } & h(x) \leqq 0, x \in X .
\end{array}
$$

Assume that $f_{j}(x) \geqslant 0, g_{j}(x)>0,1 \leqslant j \leqslant k, \forall x \in X$.

Definition 3.1: $x^{*}$ is said to be an efficient solution of (FP) if it is feasible for (FP) and there exists no other feasible solution $x$ of (FP) such that

$$
\frac{f(x)}{g(x)} \leqslant \frac{f\left(x^{*}\right)}{g\left(x^{*}\right)} .
$$

DEFINITION 3.2: $x^{*}$ is said to be a properly efficient solution of (FP) if it is efficient for (FP) and if there exists a scalar $M>0$ such that, for each $i$,

$$
\frac{\left(f_{i}\left(x^{*}\right) / g_{i}\left(x^{*}\right)\right)-\left(f_{i}(x) / g_{i}(x)\right)}{\left(f_{j}(x)\right) /\left(g_{j}(x)\right)-\left(f_{j}\left(x^{*}\right)\right) /\left(g_{j}\left(x^{*}\right)\right)} \geqq M
$$

for some $j$ such that $\left(f_{j}(x)\right) /\left(g_{j}(x)\right)>\left(f_{j}\left(x^{*}\right)\right) /\left(g_{j}\left(x^{*}\right)\right)$ whenever $x$ is feasible for (FP) and $\left(f_{i}(x)\right) /\left(g_{i}(x)\right)<\left(f_{i}\left(x^{*}\right)\right) /\left(g_{i}\left(x^{*}\right)\right)$.

Following Bector's parametric approach reported in [1], we consider the following multiobjective optimisation problem.

Primal Problem $\left(M P_{v}\right)$ :

$$
\begin{array}{ll}
\text { Minimise } & \left(f_{1}(x)-v_{1} g_{1}(x), \ldots, f_{k}(x)-v_{k} g_{k}(x)\right) \\
\text { subject to } & h(x) \leqq 0, x \in X .
\end{array}
$$

The following Lemma can be proved using similar lines to that given in Kaul and Lyall [7]. 
LEMMA 3.1. Let $x^{*}$ be a properly efficient solution of (FP). Then, $x^{*}$ is a properly efficient solution of $\left(M P_{v^{*}}\right)$ with $v^{*}=\left(f\left(x^{*}\right)\right) /\left(g\left(x^{*}\right)\right)$.

Conversely if $x^{*}$ is a properly efficient solution of $\left(M P_{v^{*}}\right)$ where $v_{j}^{*}=\left(f_{j}\left(x^{*}\right)\right) /\left(g_{j}\left(x^{*}\right)\right)$, $j=1,2, \ldots, k$, then $x^{*}$ is a properly efficient solution of (FP).

Following Geoffrion's idea reported in [5], we consider the scalar programs corresponding to $(F P)$ and $\left(M P_{v^{*}}\right)$.

Primal Problem $(F P)_{\alpha^{*}}$ :

$$
\begin{aligned}
& \text { Minimise } \alpha^{* T}\left(\frac{f(x)}{g(x)}\right) \\
& \text { subject to : } \quad h(x) \leqq 0, x \in X .
\end{aligned}
$$

Primal Problem $\left(M P_{v^{*}}\right)_{\alpha^{*}}$ :

$$
\begin{aligned}
& \text { Minimise } \quad \alpha^{* T}\left(f(x)-v^{*} g(x)\right) \\
& \text { subject to : } \quad h(x) \leqq 0, x \in X .
\end{aligned}
$$

The following results are due to Geoffrion [5].

LEMMA 3.2. If $x^{*}$ is an optimal solution of $(F P)_{\alpha}$ for some $\alpha^{*} \in \mathbb{R}^{k}$ with strictly positive components, then $x^{*}$ is a properly efficient solution of (FP).

LEMMA 3.3. If $x^{*}$ is an optimal solution of $\left(M P_{v}\right)_{\alpha}$ for some $\alpha^{*} \in \mathbb{R}^{k}$ with strictly positive components, then $x^{*}$ is a properly efficient solution of $(F P)$.

The following Lemma shows that the converse of the result reported Lemma 3.2 is valid under semipreinvexity.

LEMMA 3.4. If $x^{*}$ is a properly efficient solution of (FP), and $f_{i},-g_{i}, i=1,2, \ldots, k$, and $h_{j}, j=1,2, \ldots, m$, are semipreinvex functions with respect to a same $\eta$ on $X$, then $x^{*}$ is an optimal solution of $\left(M P_{v^{*}}\right)_{\alpha^{*}}$, where $v_{j}^{*}=\left(f_{j}\left(x^{*}\right)\right) /\left(g_{j}\left(x^{*}\right)\right), j=1,2, \ldots, k$, and

$$
\alpha^{*} \in \alpha^{+}=\left\{\alpha \in \mathbb{R}^{k}: \alpha>0, \sum_{i=1}^{k} \alpha_{i}=1\right\} .
$$

Proof: Let $x^{*}$ be a properly efficient solution of (FP), then $x^{*}$ (by Lemma 3.1) is a properly efficient solution of $\left(M P_{v^{*}}\right)$, where $v_{j}^{*}=\left(f_{j}\left(x^{*}\right)\right) /\left(g_{j}\left(x^{*}\right)\right), j=1,2, \ldots, k$. Since $f_{i},-g_{i}, i=1,2, \ldots, k$, and $h_{j}, j=1,2, \ldots, m$, are semipreinvex functions with respect to the same $\eta$, it follows that $f_{i}-v_{i}^{*} g_{i}$ is a semipreinvex function with respect to the same $\eta$ for each $i=1,2, \ldots, k$. Using a similar proof to [10, Theorem 3.2], we see that $x^{*}$ is an optimal solution of $\left(M P_{v^{*}}\right)_{\alpha^{*}}$ where $\alpha^{*} \in \alpha^{+}$.

Now we define the vector saddle point Lagrangian for (FP) as follows:

$$
\phi(x, y)=\frac{f(x)+y^{T} h(x) e}{g(x)}=:\left(\frac{f_{1}(x)+y^{T} h(x)}{g_{1}(x)}, \ldots, \frac{f_{k}(x)+y^{T} h(x)}{g_{k}(x)}\right)
$$


where $e=(1,1, \ldots, 1) \in \mathbb{R}^{k}$.

The vector saddle point problem for (FP) is the problem of finding $x^{*} \in X, y^{*} \in \mathbb{R}^{m}$, $y^{*} \geqq 0$ such that

$$
\begin{gathered}
\frac{f\left(x^{*}\right)+y^{T} h\left(x^{*}\right) e}{g\left(x^{*}\right)} \nsucceq \frac{f\left(x^{*}\right)+y^{* T} h\left(x^{*}\right) e}{g\left(x^{*}\right)} \\
\frac{f\left(x^{*}\right)+y^{* T} h\left(x^{*}\right) e}{g\left(x^{*}\right)} \ngtr \frac{f(x)+y^{* T} h(x) e}{g(x)}
\end{gathered}
$$

for all $x \in X, y \in \mathbb{R}^{m}, y \geqq 0$.

THEOREM 3.1. If $\left(x^{*}, y^{*}\right)$ is a solution of the vector saddle point problem and $f,-g$ and $h$ are semipreinvex with respect to a same $\eta$, then $x^{*}$ is properly efficient for $(F P)$.

Proof: Clearly, (1) implies that $h\left(x^{*}\right) \leqq 0$, or else (1) can be violated by appropriately making a component of $y$ infinitely large in magnitude. Now taking $y=0$ in (1) yields: $y^{* T} h\left(x^{*}\right) \geqq 0$. Noting that $y^{*} \geqq 0$ and $h\left(x^{*}\right) \leqq 0$ imply that $y^{* T} h\left(x^{*}\right) \leqq 0$, we have $y^{* T} h\left(x^{*}\right)=0$. Hence, $x^{*}$ is a feasible solution of (FP). (2) is equivalent to the following statement: The inequality

$$
\begin{aligned}
\left(f_{1}(x)+y^{* T} h(x)-\frac{f_{1}\left(x^{*}\right)+y^{* T} h\left(x^{*}\right) e}{g_{1}\left(x^{*}\right)} g_{1}(x),\right. \\
\left.\quad \ldots, f_{k}(x)+y^{* T} h(x)-\frac{f_{k}\left(x^{*}\right)+y^{* T} h\left(x^{*}\right) e}{g_{k}\left(x^{*}\right)} g_{k}(x)\right) \leqq 0
\end{aligned}
$$

has no solution on $X$. From the fact that $f,-g$ and $h$ are semipreinvex with respect to the same $\eta$, it follows from [11, Lemma 1] that we can find scalars $\alpha_{i}^{*}>0, i=1,2, \ldots, k$, such that

$$
\sum_{i=1}^{k} \alpha^{*} \frac{f_{i}\left(x^{*}\right)}{g_{i}\left(x^{*}\right)}+\sum_{i=1}^{k} \alpha^{*} \frac{y^{* T} h\left(x^{*}\right)}{g_{i}\left(x^{*}\right)} \leqq \sum_{i=1}^{k} \alpha^{*} \frac{f_{i}(x)}{g_{i}(x)}+\sum_{i=1}^{k} \alpha^{*} \frac{y^{* T} h(x)}{g_{i}(x)}
$$

for all $x \in X$.

The equality $y^{T} h\left(x^{*}\right)=0$ and (3) give that

$$
\sum_{i=1}^{k} \alpha^{*} \frac{f_{i}\left(x^{*}\right)}{g_{i}\left(x^{*}\right)} \leqq \sum_{i=1}^{k} \alpha^{*} \frac{f_{i}(x)}{g_{i}(x)}
$$

for a feasible solution $x$ of $(F P)$. Then $x^{*}$ is an optimal solution of $(F P)_{\alpha^{*}}$. Thus, by Lemma 3.1, it follows that $x^{*}$ is a properly efficient solution of (FP).

The program (FP) will be said to satisfy the generalised Slater constraint qualification if $h$ is semipreinvex with respect to $\eta$ and there exists $x_{1} \in X$ such that $g\left(x_{1}\right)<0$.

THEOREM 3.2. Let $x^{*}$ be a properly efficient solution for (FP). If the generalised Slater's constraint qualification is satisfied, and $f,-g$ and $h$ are semipreinvex with respect 
to a same $\eta$, then there exists a $y^{*} \geqq 0$ such that $\left(x^{*}, y^{*}\right)$ is a solution of the vector saddle point problem.

Proof: Since $x^{*}$ is a properly efficient solution of (FP), it is also an optimal solution of $\left(M P_{v^{*}}\right)_{\alpha^{*}}$, by Lemma 3.4, where $v_{j}^{*}=\left(f_{j}\left(x^{*}\right)\right) /\left(g_{j}\left(x^{*}\right)\right), j=1,2, \ldots, k$, and $\alpha^{*} \in \alpha^{+}$. Since $\alpha^{* T}\left(f-v^{*} g\right)$ is semipreinvex on $X$ with respect to $\eta$, it can be shown by following an similar line to that given for $\left[10\right.$, Theorem 4.1] that there exists a $y^{*} \in \mathbb{R}^{m}, y^{*} \geqq 0$ such that $y^{* T} h\left(x^{*}\right)=0$ and

$$
L\left(x^{*}, y\right) \leqslant L\left(x^{*}, y^{*}\right) \leqslant L\left(x, y^{*}\right)
$$

for all $x \in X, y \in \mathbb{R}^{m}, y \geqq 0$, where $L(x, y)=\alpha^{*}\left(f(x)-v^{*} g(x)+y^{T} h(x) e\right)$.

If (1) is not true, then, for some $i \in\{1,2, \ldots, k\}, \bar{y} \in \mathbb{R}^{m}, \bar{y} \geqq 0$, we have

$$
\frac{f_{i}\left(x^{*}\right)}{g_{i}\left(x^{*}\right)}+\frac{\bar{y}^{T} h\left(x^{*}\right)}{g_{i}\left(x^{*}\right)}>\frac{f_{i}\left(x^{*}\right)}{g_{i}\left(x^{*}\right)}+\frac{y^{* T} h\left(x^{*}\right)}{g_{i}\left(x^{*}\right)}
$$

and

$$
\frac{f_{j}\left(x^{*}\right)}{g_{j}\left(x^{*}\right)}+\frac{y^{* T} h\left(x^{*}\right)}{g_{j}\left(x^{*}\right)} \leqq \frac{f_{j}\left(x^{*}\right)}{g_{j}\left(x^{*}\right)}+\frac{\bar{y}^{T} h\left(x^{*}\right)}{g_{j}\left(x^{*}\right)}
$$

for all $j \neq i$.

Multiply (5) by $\alpha_{i}^{*} g_{i}\left(x^{*}\right)$ and (6) by $\alpha_{j}^{*} g_{j}\left(x^{*}\right)$ for $j \neq i$. Then, add them together. This, however, yields a contradiction to the first inequality in (4) for $y=\bar{y}$. Similarly if (2) is not true, then, for some $i \in\{1,2, \ldots, k\}, \bar{x} \in X$, we have

$$
\frac{f_{i}\left(x^{*}\right)}{g_{i}\left(x^{*}\right)}+\frac{y^{* T} h\left(x^{*}\right)}{g_{i}\left(x^{*}\right)}>\frac{f_{i}(\bar{x})}{g_{i}(\bar{x})}+\frac{y^{* T} h(\bar{x})}{g_{i}(\bar{x})}
$$

and

$$
\frac{f_{j}(\bar{x})}{g_{j}(\bar{x})}+\frac{y^{* T} h(\bar{x})}{g_{j}(\bar{x})} \leqq \frac{f_{j}\left(x^{*}\right)}{g_{j}\left(x^{*}\right)}+\frac{y^{* T} h\left(x^{*}\right)}{g_{j}\left(x^{*}\right)}
$$

for all $j \neq i$.

Multiply (7) and (8) by $\alpha_{i}^{*} g_{i}(\bar{x}), i=1,2, \ldots, k$. Then, add them together. This again yields a contradiction to the second inequality of (4) in view of the fact that $y^{* T} h\left(x^{*}\right)=0$ and $v_{i}^{*}=\left(f_{i}\left(x^{*}\right)\right) /\left(g_{i}\left(x^{*}\right)\right), i=1,2, \ldots, k$. Thus, (1) and (2) hold. Therefore, $\left(x^{*}, y^{*}\right)$ is a solution of the vector saddle point problem.

\section{REFERENCES}

[1] C.R. Bector, S. Chandra and M.K. Bector, 'Generalized fractional programming duality: a parametric approach', J. Optim. Theory Appl. 60 (1989), 243-260.

[2] B.D. Craven, 'Invex functions and constrained local minima', Bull. Austral. Math. Soc. 24 (1981), 357-366. 
[3] B.D. Craven and B. Mond, 'Fractional programming with invexity', in Progress in optimization, Appl. Optim. 30 (Kluwer Acad. Publ., Dordrecht, 1999), pp. 79-89.

[4] M.A. Hanson, 'On sufficiency of the Kuhn-Tucker conditions', J. Math. Anal. Appl. 80 (1981), 544-550.

[5] A.M. Geoffrion, 'Proper efficiency and the theory of vector maximization', J. Math. Anal. Appl. 22 (1968), 618-630.

[6] Z.A. Kahn and M.A. Hanson, 'On ratio invexity in mathematical programming', $J$. Math. Anal. Appl. 205 (1997), 330-336.

[7] R.N. Kaul and V. Lyall, 'A note on nonlinear fractional vector maximization', Opsearch 26 (1989), 108-121.

[8] M.A. Noor, 'Nonconvex function and variational inequalities', J. Optim. Theory Appl. 87 (1995), 615-630.

[9] T. Weir and V. Jeyakumar, 'A class of nonconvex functions and mathematical programming', Bull. Austral. Math. Soc. 38 (1988), 177-189.

(10] T. Weir and B. Mond, 'Pre-invex functions in multiple objective optimization', J. Math. Anal. Appl. 136 (1988), 29-38.

[11] X.Q. Yang and G.Y. Chen, 'A class of nonconvex functions and pre-variational inequalities', J. Math. Anal. Appl. 169 (1992), 359-373.

Current address:

Department of Applied Mathematics

The Hong Kong Polytechnic University

Hung Hom, Kowloon

Hong Kong

e-mail: maxmyang@polyu.edu.hk

and

Department of Mathematics

Chongqing Normal University

Chongqing 40047

China
Department of Applied Mathematics

The Hong Kong Polytechnic University

Hung Hom, Kowloon

Hong Kong, China

Department of Applied Mathematics

The Hong Kong Polytechnic University

Hung Hom, Kowloon

Hong Kong, China 